\title{
Socioeconomic status and stress: Neuroendocrine pathways to disease
}

\author{
Soma Hewa \\ School of Policy Studies, Queen's University, Kingston, Ontario, Canada, and School of Public Administration, \\ Guangzhou University, Guangzhou, China.
}

Correspondence: Professor Soma Hewa

e-mail: hewa@queensu.ca

\begin{abstract}
The biomedical model investigates diseases of the organism at the cellular level, and ignores the broader socioeconomic factors that affect health. This discursive analysis examines the physiological mechanism by which socioeconomic factors get inside the human body and make people susceptible to diseases. A survey of recent materials was carried out using PubMed database to identify peer-reviewed manuscripts that examine physiological reaction to stress and its connection to diseases. These studies reveal a complex relationship between socioeconomic status and chronic diseases.
\end{abstract}

Key words: Stress, adaptation, allostasis, allostaticload, illness

\section{Introduction}

During the last three decades, researchers have been able to establish the physiological connection between socioeconomic status and diseases such as hypertension, coronary heart diseases, stroke, type II diabetes, memory impairment, and malignancies, which seem to increase as we go down the socioeconomic ladder of a society. While the social gradient may explain the continuous distribution of risks and vulnerabilities, the fundamental question that these researchers have been trying to answer was how does socioeconomic status get inside our bodies and make us susceptible to diseases? This discursive analysis, based on the current literature, explains the physiological mechanism through which socioeconomic status manifests as a major cause of disease, and makes some policy recommendations to mitigate the socioeconomic challenges, and their health damaging consequences.

\section{Understanding the social determinants of health}

The social determinants of health include a wide range of interrelated socioeconomic factors that affect people's everyday living. For example, income and employment status, the level of education, early childhood experience, and access to healthcare are some of the important social and economic factors that affect health throughout life. Yet, almost universally, these factors are not adequately incorporated into health policies and practices. Health has long been regarded as an outcome of good medicine. Although modern medical breakthroughs have eradicated many infectious diseases, the level of exposure to disease risks is often dependent upon a wide range of socioeconomic factors. It is only recently that policy makers have begun to examine population health within the broader context of socioeconomic status, and to introduce population health promotion strategies as part of the overall socioeconomic policies.

The social and economic problems influence health in every stage of life. For example, recent studies have shown that low birth weight and emotional deprivation in childhood are major causes of learning disabilities and other behavioral problems, which could in turn be a precursor to long-term material disadvantages in adult life. Moreover, 
these material disadvantages are both absolute and relative: for example, not having money to buy food, or a place to live is a measure of an absolute poverty, which is a common problem among the people of the lowest rung of the social hierarchy. The effect of absolute poverty during in-utero growth through early childhood is known to produce biological markers for many adulthood diseases, such as diabetes and coronary artery diseases (1).

Relative poverty, on the other hand, is the difference between the standard of living enjoyed by the people in upper social echelons and those in the lower social classes in the same society. Relative poverty is believed to affect health through psychological and other status symbols. Although absolute poverty has significantly declined in recent years, relative poverty is rising throughout the world due to widening income gap between the rich and the poor. Studies have shown that in societies where socioeconomic disparities are higher, the overall mortality rate, infant mortality, violent crimes, hostility, obesity, and interpersonal distrust are greater (2). Thus, health is powerfully affected by both absolute and relative socioeconomic status. As we go down the social ladder within a given society, morbidity and mortality rates increase because the same people suffer from both types of deprivation. How does socioeconomic status get "under the skin," and make people sick?

\section{Adaptation to socioeconomic pressure}

The link between socioeconomic status and disease is "stress," the term that often used to express the physical and psychological "pressure" exerted by various external challenges. These external challenges, which include socioeconomic problems, are powerful stressors in life. However, the impact of these stressors varies depending on the length of exposure to such challenges, the availability of supportive resources, and the individual genetic factors. Bruce McEwen defines stress as "a threat, real or implied, to the psychological or physiological integrity of an individual" (3). He recognizes, however, the ambiguity of the term as it is used in everyday life, which makes it difficult for us to understand how the human body copes with stress. As researchers have pointed out in recent years, this coping mechanism, which is described as allostasis, enables the organism to adapt to the external challenges, so that it can maintain the internal functional balance (homeostasis) of organ systems, and sustain life. Any physical or psychological challenge disturbs the functional balance of organ systems, and the body reestablishes it by adjusting neuroendocrine adaptation. Humans have survived on the earth by adapting to their physical and social environments. Overtime, however, the coping mechanism increases the vulnerability to major diseases, which is described as the allostatic load, or the cumulative wear and tear on the organ systems and their tissues due to prolong, insufficient, or failed adaptation (4).

As the brain determines the nature of the challenge, it regulates neuroendocrine response. Coping with any acute challenge requires extra energy for physical and psychological endurance. Thus, the autonomic nervous system, via the hypothalamicpituitary-adrenal (HPA) axis, triggers the catabolic function of the digestive system resulting in an increase release of energy by breaking down protein, carbohydrate, and fat storages in the muscles and the liver. The release of three major endocrines (hormones), such as epinephrine, norepinephrine, and dopamine (collectively known as catecholamine) from the adrenal medulla increases the supply of blood to the heart, brain, and skeletal muscles. This in turn triggers fundamental physiological reactions affecting blood pressure: 1) the release of epinephrine triggers the discharge of the glucagon hormone by the pancreas to breakdown glycogen, the energy storages in the liver, which increases the plasma levels of glucose, free fatty acids, low-density lipoprotein, and cholesterol to provide more energy; 2) the discharge of norepinephrine stimulates the heart muscles to increase cardiac output (the heartbeat), and vasoconstrictions (tightening of the arteries) to eject more blood to the brain and skeletal muscles, and less blood to the gut and kidney; 3) norepinephrine stimulates the release of renin-aldosterone hormone in the kidney, which increases the plasma sodium concentration and arterial blood pressure. The renin-aldosterone mechanism increases the retention of sodium fluids in the blood flow, and prevents releasing the liquid through urine; 4) dopamine, primarily a neurotransmitter from the brain and autonomic 
nervous system, regulates the blood flow through the arteries, and the secretion of both epinephrine and norepinephrine. With the rising cardiac output, fluid volume, and vasoconstriction, the blood pressure rapidly elevates forcing more blood to the brain and skeletal muscles, while restricting the blood flow to elsewhere in the body (5).

As these neuroendocrine mechanisms intensify the functional output of several organ systems, the release of the glucocorticoid hormone from the adrenal cortex regulates the catabolic process that increases the blood level of glucose. Obviously, all these physiological and neuroendocrine reactions to an external challenge require a fundamental shift in the performance of a number of major organ systems from an anabolic state of repairing tissues, producing immune cells, stimulating reproductive hormones, and storing excess energy to a catabolic state in which more energy is provided to certain organ systems to intensify their activity, while suppressing the performance of others temporarily in order to sustain life (or to face the challenge).

\section{Adaptation and disease}

The stress induced disease, or allostatic load, is the chronic wear and tear on the body resulting from adaptation to stressor. As noted, a common outcome of the adaptation (allostasis) is cardiovascular reactivity resulting in high blood pressure. The elevated blood pressure accompanies several risk factors such as increased blood levels of glucose, low-density lipoprotein, and cholesterol, which are all known clinical risk factors for heart diseases, diabetes, obesity, and strokes. Studies have shown that once the neuroendocrine process raises the blood pressure, and maintains it at a higher level for an extended period, blood pressure tends to stay high even if the initial cause of the elevation no longer exists. The brain and arteries develop structural and functional remodelling as part of the adaptation. This remodelling, over time, particularly in connection with arterial constriction, causes damage in the inner lumen of the arterial walls, where atherosclerotic plaques begin to develop, obstructing the blood flow. At this stage, even if the fluid volume has become normal, the narrowed arteries with blockages are bound to cause heart attacks and strokes (6).
Also, the release of glucocorticoid hormone during stress undermines the production of white blood cells, which has several pathological consequences. During prolonged stress the suppressed immune system delays the healing of wounds and injuries, and most importantly exposes the organism to various pathogenic agents such as viruses, bacteria, and carcinogenic agents, which are normally removed from the body by white blood cells. The immune system, particularly the thymus gland and its ability to produce white blood cells, is mediated by glucocorticoids (7).

Likewise, under chronic stress, glucocorticoid impedes the insulin function that regulates the blood glucose level. The function of insulin is to remove the extra glucose from the blood and store them in the muscles, body fat, and the liver as glycogen to be utilized when the blood glucose level goes down. During stress, the increased demand for energy to meet an external challenge prevents this insulin activity resulting in abnormally high levels of blood glucose. While elevating blood levels of glucose and free fatty acids, and preventing insulin from storing them in the muscles and the liver, the glucocorticoid promotes the deposition of glycogen in the abdomen. It has been identified that abdominal obesity, as in Cushing's disease, as well as type I and II diabetes are triggered by chronic stress (8). A number of recent studies have substantiated these observations reporting that excessive metabolic disorders among low-income groups, such as African-Americans, are related to psychosocial stress of living in hierarchical societies (9). Kate Pickett and colleagues found that in the top 50 developed countries, adjusting for gross national per capita income, the distribution of income is positively correlated with a number of health indicators such as the percentage of obese people in the population, diabetes-related mortality rate, and the average daily per capita calories intake (10).

While the brain is the main organ that regulates the neuroendocrine response to stress, it is also subjected to major neurological remodeling and structural transformations due to prolonged stress. Both animal and human studies have shown that prolonged activation of glucocorticoid receptors of the brain increases the risk of protein plaques deposition on the neurons obstructing the chemical messengers (neurotransmitters). The increased blood flow to the 
brain also carries with them high levels of plasma protein that accumulates on the neurons over time, eventually destroying them altogether. The amyloid hypothesis suggests that the accumulation of amyloid plaques on neurons may be linked to Alzheimer's disease. Recent studies of type II diabetes, which is connected to increased plasma level of glucocorticoid under chronic stress, found reduced hippocampal volume (shrinking of the hippocampus). They have also shown that the reduced hippocampal volume is associated with memory impairment and cognitive dysfunction, and a general relationship between the size of the hippocampus and spatial memory (11). Studies based on human neuroimaging have shown that prolonged stress and post-traumatic stress disorders produce drastic structural changes in the prefrontal cortex, hippocampus, and amygdala leading to chronic depressive symptoms and anxiety disorders (12).

The neuroendocrine reactions triggered by socioeconomic challenges inevitably impose undue pressure on the organ systems and their tissues in the long-term. Generally, it is constructive when the neuroendocrine reactivity is rapidly mobilized on the organ systems and terminated immediately. However, when the neuroendocrine reactivity is prolonged due to chronic stress, it undermines mental and physical health. How rapidly those neuroendocrine mechanisms are activated and are halted is dependent upon the availability of effective coping mechanisms and resources for individuals, families, and communities. Unhealthy coping mechanisms could further aggravate damaging health consequences.

\section{Behavioural adaptations as coping mechanisms}

The pathophysiological conditions resulting from the adaptation to stress are often exacerbated by a variety of harmful behavioural adaptations, such as smoking, drinking, overindulging, and the lack of physical activity, which are often perceived as coping strategies by some individuals. These behavioural adaptations have the same pathogenic outcomes for major organ systems, as do the physiological adaptions to stress.
By contrast, however, studies have shown that the availability of social support, trusting interpersonal relations, regular physical activity, and membership in community organizations, which have been collectively described as social capital, enhances the resilience of organ systems. Any supportive relationship that has an attenuating effect on psychophysiological stress may have a positive long-term impact on health (13). People with social networks receive both material and emotional support during stressful situations in their lives. Social connectivity and supportive environments are known to increase inhibitory signals to the HPA axis, which in turn raises the HPA activation threshold thereby minimizing the effect of potential socioeconomic challenges to physiological system. In other words, if the individual is confident that support is available at a time of "distress" such knowledge could prevent the full-blown stress response from the HPA axis. The individual differences in social integration and connectivity (social networks) could therefore modify the cognitive perceptions of external challenges, which may in turn moderate the neuroendocrine reactions to such challenges (14).

\section{Conclusion; policy recommendations}

In recent years, particularly in developed Western countries, public policies on population health promotion have increasingly been incorporated into major economic policies. There has been significant effort to reduce the social gradient in health through inclusive economic policies, which have the potential to increase prosperity, greater redistribution of resources, and the overall social cohesiveness. These policies are results of several well-recognized public inquiries into the impact of socioeconomic inequalities in some countries, and the global campaigns by various multilateral organizations, such as the World Health Organization (WHO) and the World Bank, to inform about the "health cost" of inequality and economic marginalization. For example, the report of the Donald Acheson Commission of the United Kingdom, and the WHO Commission on Social Determinants of Health advocate that population health strategies to be based on four major pillars to counter the potential impact of material 
disadvantages from early childhood through adulthood: 1) reducing poverty and income inequality through targeted redistributive welfare policies to give greater educational and employment opportunities for disadvantaged social groups; 2) preventing unhealthy life-styles and life course issues, such as smoking, drinking, and malnutrition to promote healthy development; 3) prohibiting discrimination based on age, gender and ethnicity in areas such as employment, education and housing to ensure social justice and equality; 4) ensuring universal access to quality healthcare, particularly primary care (15).

The underlying philosophy that guides these broad policy recommendations is that good health for all can be achieved by providing a good "foundation" in life through guaranteed access to education, healthcare and employment. Life is a progression through stages, in that material disadvantages in early childhood that prevent getting a good education, undermines the labour market opportunities during adulthood. People who have experienced disadvantages in early childhood are at the greatest risk in the subsequent stages of life. Policies need to prevent people from experiencing disadvantages at the earliest possible stages of life, and they need to give priority to those who are already at a disadvantage in society. These are the people who suffer from both absolute and relative deprivations.

The social environments-at work, school, or neighbourhood-that provide a sense of belongingness, security and safety are known as "healthy environments" as they prevent marginalization and deprivation, and promote a sense of well-being. In such social environments, individuals are less likely to make unhealthy lifestyle choices. By contrast, environments, in which people feel excluded, abused and discriminated, are regarded as "toxic environments," where people feel less worthy of themselves, seek the comfort of health damaging behaviours, and engage in criminal activities. Therefore, national and local authorities, as well as public and private employers, and community leaders need to recognize the health implication of social integration in institutions, as well as, in communities and neighbourhoods. Policies that prevent discrimination, abuse and violence not only increase economic productivity, but also promote health and well-being. People who are more socially connected: 1) live longer; 2) are more likely to survive a myocardial infarction; 3 ) are less likely to experience a recurrence of cancer; 4) are less likely to suffer from infectious illness, than those who are less integrated to the community (16).

Likewise, access to healthcare is a basic human right that has been recognized by the World Health Organization. Specifically, the access to primary care is critical for preventing disease, immunizing children and providing prenatal care services that build the foundation for healthy living. Although health care services themselves focus on the clinical aspects of risks rather than the social determinants of exposure to risks, preventive health services protect people from diseases and premature death. Public policies must ensure that all citizens have guaranteed access to essential health services, so that an unforeseen illness would not prevent people from fully participating in education and employment resulting in life-long socioeconomic disadvantage. Public policies need to address the socioeconomic determinants of health before they manifest as major health problems. This is the challenge for both policy makers and political leaders.

\section{References}

1. Barker DJP (1990). Fetal and infant origins of adult disease. British Medical Journal, 301: 1111: Danese A and McEwen BS (2012). Adverse childhood experiences, allostasis, allostatic load, and age-related disease. Physiology \& Behavior, 106: 29-39: Barker DJP, Meade TW, Fall CHD, Lee A, Osmond C, Phipps K, and Stirling Y (1992) Relation of fetal and infant growth to plasma fibrinogen and factor VII concentrations in adult life, British Medical Journal, 304:148-152.

12. Kawachi I, Kennedy BS, Wilkinson RG (1999). Crime: social disorganization and relative deprivation. Social Science and Medicine, 48: 719-31; Marmot M (2012). Health equity: the challenge. Australian and New Zealand Journal of Public Health, 36: 513-14; Elgar FJ (2010). Income inequality, trust, and population health in 33 countries. American Journal of Public Health, 100: 23112315.

3. McEwen BS (2000). The neurobiology of stress: from serendipity to clinical relevance. Brain Research, 886: 172-189. 
4. Sterling P, Eyer J (1981). Biological basis of stress-related mortality. Social Science and Medicine, 15: 3-42; Sapolsky MR (2004). Social status and health in humans and other animals. Annual Review of Anthropology, 33: 393-418; McEwen BS, (2006). Protective and damaging effects of stress mediators: central role of the brain, Dialogues in Clinical Neuroscience, 8: 367-381; McEwen BS, (2012). Brain on stress: how the social environment gets under the skin, Proceedings of the National Academy of Science, 109 (Suppl. 2): 17180-17185.

5. Tobian L (1972). A viewpoint concerning the enigma of hypertension. The American Journal of Medicine, 52: 595 609; McEwen BS (2003). Interacting mediators of allostasis and allostatic load: towards an understanding of resilience in aging, Metabolism, 52: 10-16.

6. McEwen BS, Gianaros PJ (2010). Central role of the brain in stress and adaptation: links to socioeconomic status, health, and disease, Annals of New York Academy of Science, 1186: 190-222; Sterling P, Eyer J, (1988) Allostasis: a new paradigm to explain arousal pathology, In: Fisher S, Reason J (Eds.) Handbook of Life stress, cognition and health, Wiley, New York, pp.631-651.

7. Cohen S, Adler N, Alper CM, Doyle WJ, Treanor JJ (2008). Objective and subjective socioeconomic status and susceptibility to the common cold. Health Psychology, 27: 268-274; Dowdell K, Whitacre C (2000). Coping with the environment: Neural and endocrine mechanisms. Oxford University Press, New York.

8. Goodman E, Adler N, Daniels ER, Morrison JA, Slap GB, Dolan LM (2003). Impact of objective and subjective social status on obesity in a biracial cohort of adolescents, Obesity Research, 11: 1018-1026.

9. Carlson ED, Chamberlain RM (2005) “Allostatic load and health disparities: A theoretical orientation, Research in Nursing \& Health, 28: 306-315; Wilkinson RG, Pickett KE (2007). The problem of relative deprivation: why some societies do better than others, Social Science and Medicine, 65: 1965-1978; Brunner EJ, Chandola T, Marmot MG (2007). Prospective effect of job strain on general and central obesity in the Whitehall II Study, American Journal of Epidemiology, 165: 828-837; Pickett KE, Wilkinson RG (2015). Income inequality and health: a causal review, Social Science \& Medicine, 128: 316-326.

10. Pickett KE, Kelly S, Brunner E, Lobstein T, Wilkinson RG (2005). Wider income gaps, wider waist-bands? an ecological study of obesity and income inequality, Journal of Epidemiology and Community Health, 59: 670-674.
11. de Felice FG (2013). Alzheimer's disease and insulin resistance: translating basic science into clinical applications, The Journal of Clinical Investigation, 123: 531-539; McEwen BS, Gray JD, and Nasca C, (2015). Recognizing resilience: learning from the effects of stress on the brain. Neurobiology of Stress, 1: 1-11.

12. Convit A, Wolf OT, Tarshish C, de Leon MJ (2003). Reduced glucose tolerance is associated with poor memory performance and hippocampal atrophy among normal elderly, Proceedings of the National Academy of Science, 100: 2019-2022; Sapolsky MR (1996). A baboon's life: Male-on-male violence, aging and friendship on the savannah, Stanford Today, July/August, 49-51.

13. Berkman LF, Glass T (2000). Social integration, social networks, social support, and Health, In: Berkman LF, Kawachi I (Eds.) Social Epidemiology. Oxford University Press, New York, pp. 137-173; Hamer M (2012). Psychosocial stress and cardiovascular disease risk: The role of physical activity, Psychosomatic Medicine, 74: 896-903.

14. Kawachi I, Kim D, Coutts A, Subramanian SV (2004). Commentary: Reconciling the three accounts of social capital, International Journal of Epidemiology, 33: 682690; Carpiano RM (2007). Neighbourhood social capital and adult health: An empirical test of Bourdieu-based model, Health and Place, 13: 693-655.

15. Acheson D (1998). Commission of the United Kingdom, Independent inquiry into inequalities in health report, Department of Health, The Stationery Office, United Kingdom; World Health Organization, 2008 Closing the gap in a generation, World Health Organization's Commission on Social Determinants of Health. Geneva; World Bank, 2016 Poverty and shared prosperity: taking on inequality, Washington DC.

16. Prus SG (2011). Comparing social determinants of selfrated health across the United States and Canada, Social Science \& Medicine, 73: 50-59; Kawachi I, Kennedy BS, Wilkinson RG (1999) Crime: social disorganization and relative deprivation, Social Science and Medicine, 48: 719-731; Carpiano RM, Fitterer LM (2014). Questions of trust in health research on social capital: what aspects of personal network social capital do they measure? Social Science and Medicine, 116: 225-234. 